
dures d'origine animale

\title{
Trésors de la Terre
}

L'exposition minéralogique du Muséum national d'histoire naturelle (Paris)

\section{Catherine Cardinal et Cristiano Ferraris}

\section{(2) OpenEdition}

\section{Journals}

Édition électronique

URL : http://journals.openedition.org/artefact/1615

DOI : 10.4000/artefact. 1615

ISSN : 2606-9245

Éditeur :

Association Artefact. Techniques histoire et sciences humaines, Presses universitaires du Midi

Édition imprimée

Date de publication : 30 mai 2018

Pagination : 253-263

ISBN : 978-2-7535-7494-6

ISSN : 2273-0753

Référence électronique

Catherine Cardinal et Cristiano Ferraris, «Trésors de la Terre », Artefact [En ligne], 7 | 2017, mis en ligne le 14 février 2019, consulté le 15 septembre 2020. URL : http://journals.openedition.org/artefact/1615

\section{c) (i)}

Artefact, Techniques, histoire et sciences humaines est mise à disposition selon les termes de la Licence Creative Commons Attribution - Pas d'Utilisation Commerciale - Pas de Modification 4.0 International. 


\section{Trésors de la Terre L'exposition minéralogique du Muséum national $d$ 'histoire naturelle (Paris)}

Catherine CARDINAL Cristiano FERRARIS**

\section{Science et art réunis pour informer, séduire}

L'exposition Trésors de la Terre, présentée dans l'aile droite de la Galerie de minéralogie et de géologie au Jardin des Plantes de Paris, offre une approche à la fois scientifique et esthétique du monde des minéraux. Les quelque six cents spécimens minéralogiques présentés, allant de cristaux géants aux gemmes de la Couronne de France, reflètent sa diversité, sa complexité et sa richesse artistique. S'appuyant sur des minéraux bruts très variés, souvent rares, des pierres précieuses exceptionnelles, des bijoux et des objets d'art fameux, le parcours muséographique exprime clairement le discours des auteurs de l'exposition. Le dessein de Cristiano Ferraris, commissaire et responsable des collections de minéralogie du Muséum $d^{\prime}$ 'histoire naturelle (MNHN), d'initier le public aux aspects divers des minéraux, qu'il s'agisse de leur formation, de leur développement et de leurs utilisations, est pleinement atteint.

\section{Le choix de spécimens et d'objets précieux}

La réunion d'un tel ensemble a été permise par les collections du MNHN, sans cesse augmentées jusqu'à nos jours ${ }^{1}$. Des pièces sont ainsi issues des trésors de la Couronne, des confiscations effectuées sous la Révolution, des achats de grandes collections (Weiss, Haüy, Vésignié, Deleff), des donations du tsar Nicolas ${ }^{\mathrm{er}}$, des dons du richissime industriel John Pierpont Morgan et, depuis 1990, de la Fondation Total.

\footnotetext{
*. Catherine Cardinal est professeure en histoire de l'art à l'université Clermont-Auvergne. Contact : [catherine.cardinal@wanadoo.fr].

** Cristiano Ferraris est maître de conférences et conservateur de la collection de minéraux et gemmes de la Galerie de minéralogie, Institut de minéralogie, de physique des matériaux et de cosmochimie (IMPMC), Muséum national d'histoire naturelle de Paris. Contact : [ferraris@mnhn.fr].
} 
Dans les premières sections présentant les propriétés physiques des minéraux et leur classification en dix groupes fondée sur la composition chimique qui les caractérise, de nombreux et remarquables spécimens proviennent de dons faits par l'entreprise Total. Par exemple, on observe une pièce appartenant à la classe des carbonates (Fig. XXXIII, cahier couleur), composée de roses d'azurite sur malachite, découverte dans l'Utah (USA), un minéral de la classe des phosphates, pyromorphite provenant de Chine, un quartz entouré de quatre variétés de silicates, trouvé en Afghanistan (un béryl rose, un spodumène violet, un elbaïte vert bleuté, un albite bleu clair). Dans la vitrine dédiée aux métaux précieux, encore découvert par Total, un exemplaire d'or natif, reposant sur un quartz, offre un étonnant entrelacement de cristaux qui justifie son nom de "buisson d'or " (Fig. XXXIV, cahier couleur).

D'autres donations enrichissent l'exposition, particulièrement les sections dédiées aux gemmes et aux pierres ornementales. Ainsi, la collection Haüy est représentée par des cristaux taillés en améthyste et par un béryl émeraude (Fig. XXXV, cahier couleur), la donation J. Pierpont Morgan par des spécimens d'elbaïte rubellite (Fig. XXXVI, cahier couleur).

De nombreuses pièces historiques ont été réunies. Citons le béryl émeraude (Fig. XXXVII, cahier couleur) dit « de la couronne de saint Louis » (51,6 carats), le « Grand Saphir de Louis XIV » (135,8 carats) taillé en 1669, originaire du Sri Lanka, le « diamant-portrait » de l'impératrice Marie-Louise (91 carats). Cadeau de Napoléon $\mathrm{I}^{\mathrm{er}}$, en 1810, il doit son nom au fait qu'il recouvrait un portrait en miniature, situé au milieu d'un bracelet. On peut aussi remarquer l'opale en cabochon de Louis XVIII (77 carats), dotée d'une monture garnie de 48 diamants.

Des objets provenant des collections de Louis XIV rappellent le goût du roi pour les pierres dures. Sont exposés deux plateaux de table en marqueterie de pierres dures, fabriquées à Florence, ainsi que plusieurs coupes montées comme celle en jade, surmontée d'une sirène en corail, dont le pied est enrichi d'émeraudes, d'améthystes, de citrines, de turquoises et de corail $^{2}$ (Fig. XXXVIII, cahier couleur).

\section{La muséographie}

Sur le plan esthétique, l'architecture de l'exposition est une réussite. Les formes et les couleurs des minéraux sont mis en valeur dans des vitrines aux dimensions harmonieuses, dotées d'un éclairage soigneusement conçu. Une présentation aérée laisse apprécier les regroupements des pièces illustrant les différents thèmes.

La muséographie contribue à la qualité pédagogique du discours. Chaque " alcôve », clairement intitulée, est introduite par un texte concis. Des cartels permettent une identification aisée des minéraux. L'information est complétée par des écrans tactiles présentant de manière simple et convaincante les questions abordées dans la section. La curiosité et le plaisir des yeux sont habilement stimulés pour encourager le visiteur à poursuivre avec attention et patience le parcours. Son intérêt est sans cesse renouvelé d'alcôve en alcôve, l'étonnement et 
l'admiration l'engagent à examiner les sujets abordés dans chaque vitrine.

\section{La Galerie de minéralogie et de géologie}

Les origines de la Galerie de minéralogie et de géologie remontent au "Droguier du Roy », fondé par Louis XIII en mai 1626, qui réunissait des plantes médicinales, des gemmes et des minéraux aux vertus censées être curatives. Nicolas Lemery (1645-1715) publia, en 1698, un Dictionnaire universel des drogues simples, dans lequel sont énumérés les minéraux conservés dans le droguier royal. Vers 1720 , celui-ci perdit sa fonction médicinale; il prit le nom de «Cabinet royal d'histoire naturelle » au terme d'une période de réorganisation dans les décennies 1720 et 1730 . Nommé intendant du Jardin du Roi et du Cabinet, en 1739, Georges-Louis Leclerc de Buffon (1707-1788) ouvrit au public, en 1745, le cabinet qu'il augmenta par de multiples donations, notamment celles du roi de Pologne (1772), de Christian VII de Danemark (1777) et de Catherine de Russie (1777 et 1785).

Par un décret de la Convention nationale du 10 juin 1793, le Cabinet d'histoire naturelle et le Jardin du Roi furent réunis pour former le Muséum d'histoire naturelle, dont Louis Jean Marie Daubenton (1716-1800) fut le premier directeur. De nombreuses saisies révolutionnaires enrichirent alors les collections.

Édifiée, à partir de 1833, sous la direction de l'architecte Charles Rohault de Fleury, la galerie fut ouverte en 1841 au public $^{3}$. Constituée d'une nef longue de cent mètres, éclairée par des verrières, elle est traversée par deux rangées de dix- huit colonnes cannelées monumentales. De chaque côté, elle est flanquée d'une aile, précédée par un péristyle. Jusqu'en 1963, l'aile droite a abrité la bibliothèque du Muséum, ce dont témoigne l'inscription restée sur le fronton. Rénovée cette année-là, la galerie impressionnait les visiteurs par le grand nombre de minéraux exposés dans 192 vitrines verticales, 192 pupitres, par la variété des roches visibles dans 204 vitrines et 12000 tiroirs. En 2004, la galerie fut fermée; néanmoins, elle ouvrit temporairement en 2009 pour accueillir l'exposition Or des Amériques.

À partir de l'année suivante, un projet fut élaboré en vue de la présente exposition. Il avait pour cadre la salle dite " des Cristaux géants ", aménagée en 1986 dans l'aile droite de la galerie. Inauguré en juin 1987 par le Président de la République, cet espace avait été conçu pour présenter la fabuleuse acquisition d'environ quatre-vingts cristaux géants ${ }^{4}$. Dans le même temps, une salle du Trésor a été aménagée en sous-sol pour présenter les pièces les plus précieuses de la collection.

Aujourd'hui, la Galerie de minéralogie et de géologie conserve 135000 minéraux, dont 2930 gemmes taillées et objets d'art, plus de 300000 roches et une série de plus de 2000 météorites. L'exposition Trésors de la Terre ne présente donc qu'une infime partie de ses collections, mais elle permet au public de voir des pièces, la plupart exceptionnelles, dans un aménagement didactique et attrayant. En ce sens, elle est l'exemple d'une réussite parmi les nouveaux espaces muséologiques, réaménagés dans des bâtiments historiques, qui ponctuent l'actualité des musées.

C.C. 


\section{À la découverte de la minéralogie : les étapes de l'exposition}

\section{En préambule}

Le Muséum national d'histoire naturelle de Paris est dédié à la nature et à ses relations avec l'espèce humaine. L'échantillonnage de la diversité minérale de notre planète est l'une des missions du Muséum depuis 1635, lorsque les premiers minéraux sont entrés dans le «Droguier du Roi ». Résultat de près de quatre siècles d'acquisitions, de dons et de découvertes, la collection de minéralogie du MNHN comprend un ensemble systématique d'espèces minérales, de gemmes, de modèles cristallographiques, ainsi que de documents des grands scientifiques des $\mathrm{XVIII}^{\mathrm{e}}$ et XIXe siècles, comme Romé de l'Isle, Haüy, Des Cloizeaux et Lacroix.

En 2010, commence une réflexion sur une nouvelle exposition minéralogique d'environ $250 \mathrm{~m}^{2}$, située à droite de l'entrée principale de la galerie. Après plus de trois ans de discussions, l'espace est redéfini et ouvre en décembre 2014. La rénovation fut soumise aux contraintes découlant de l'utilisation d'un bâtiment historique et aux problèmes de sécurité, d'éclairage et de muséographie. L'architecture de la salle d'exposition est conçue autour d'un espace central, réservé à la présentation de cristaux géants, entouré de huit alcôves séparées (Fig. XXXIX, cahier couleur). Le parcours, tenant compte de la grande diversité des minéraux, conduit successivement les visiteurs dans ces espaces.

Différents thèmes, correspondant aux vitrines qui bordent les huit cellules, visent à présenter les minéraux, la minéralogie et la cristallographie sous divers aspects, depuis leur formation jusqu'à leur utilisation par l'homme. La visite commence par l'illustration de leurs formes innombrables, de leurs couleurs très variées et par la présentation de phénomènes étonnants, comme la fluorescence et la biréfringence, permettant au public de comprendre les caractéristiques physiques des minéraux. Dans la deuxième partie, la présentation se concentre sur la relation entre les hommes et les minéraux : les gemmes et la fascination qu'ils exercent, les pierres dures et leur usage artistique, l'histoire de la collection du MNHN et les personnalités qui l'ont créée. L'exposition se termine par un espace dédié aux météorites sélectionnées parmi les pièces les plus importantes du Muséum.

\section{Les cristaux géants}

L'espace central de l'exposition est occupé par une vingtaine de " cristaux géants " présentés dans un jeu d'ombres et de lumières mettant en valeur leur transparence et leur forme cristallographique (Fig. $\mathrm{XL}$, cahier couleur). Comme le souligne Jean-Pierre Lorand, «il est difficile de délimiter une frontière entre cristal normal et cristal géant, tant celle-ci est floue dans le Règne Minéral, contrairement au Règne Vivant où la taille maximale des individus est globalement caractéristique d'une espèce donnée $^{5}$ ». 
Pour les cristaux géants de la collection du Muséum, principalement représentés par le quartz et le feldspath, on parle de cristaux atteignant ou dépassant le mètre. Même si le quartz est l'espèce minérale la plus commune à la surface de notre planète, les chances de découvrir un cristal de quartz d'un mètre sont rares. Cette rareté illustre les conditions très spécifiques qui doivent être remplies pour permettre le " gigantisme » dans le règne minéral. Ces conditions sont la disponibilité des éléments chimiques nécessaires, l'espace, la température et le temps de croissance. Une roche très spécifique de la famille des granites, la pegmatite - $d$ 'où proviennent la plupart des cristaux géants de la collection du Muséum - rassemble généralement ces conditions.

La collection de cristaux géants a été achetée en 1982 à Ilia Deleff (1921-2012), né en Bulgarie et immigré au Brésil dans les années 1950. Parmi les spécimens les plus spectaculaires, il faut mentionner deux cristaux de quartz bi-terminés baptisés « Castor et Pollux » $635 \mathrm{~kg}$ de poids total). On peut aussi distinguer un cristal d'amazonite de $700 \mathrm{~kg}$, qui est le plus grand connu, un quartz morion $(600 \mathrm{~kg})$, deux fragments (2 tonnes et $300 \mathrm{~kg}$ ) d'un seul cristal de quartz de Golconda (Valadares, Brésil), deux cristaux de topaze de 200 et $250 \mathrm{~kg}$ chacun, un cristal hexagonal parfait de béryl $(280 \mathrm{~kg})$ et une apatite bleue $(60 \mathrm{~kg})$.

\section{Le règne minéral}

Qu'est-ce qu'un minéral? Comment les minéraux se sont-ils formés et ontils évolué depuis l'origine du système solaire, depuis l'apparition d'une atmos- phère riche en oxygène jusqu'à l'anthropisation de notre planète? Comment les scientifiques classent-ils les minéraux et pourquoi le font-ils? Ces questions fondamentales sont abordées dans une première alcôve, comme introduction à la découverte du monde minéral. Le visiteur est d'abord invité à comprendre la "genèse » des minéraux et leur évolution à travers les temps géologiques, depuis le système solaire précoce jusqu'à aujourd'hui'. Ensuite, lui sont expliqués les principes de la formation des minéraux constituant les roches.

La minéralogie de la Terre, d'autres planètes et lunes rocheuses évolue en raison de divers processus physiques, chimiques et éventuellement biologiques qui conduisent à la formation de nouvelles espèces minérales. Immédiatement après le Big-Bang, les températures étant trop élevées pour avoir des formes cristallines, les premiers éléments formés (hydrogène et hélium) étaient à l'état gazeux. À mesure que les premières étoiles se sont condensées, d'autres éléments formant des minéraux comme du carbone, de l'oxygène, du silicium et du magnésium sont apparus et certains éléments se sont probablement cristallisés. Lorsque les températures étaient encore supérieures à $1500^{\circ} \mathrm{C}$, certains microcristaux d'une douzaine d'espèces minérales ont pu se condenser, y compris des éléments natifs (diamant/graphite $C$ ), des nitrures (Nierite $\left[\mathrm{Si}_{3} \mathrm{~N}_{4}\right]$, osbornite [TiN]), des carbures (Moissanite [SiC]), des oxydes (Rutile $\left[\mathrm{TiO}_{2}\right]$ ), corindon $\left(\mathrm{Al}_{2} \mathrm{O}_{3}\right)$, spinel $\left.\left(\mathrm{MgAl}_{2} \mathrm{O}_{4}\right)\right)$ et des silicates (Forsterite $\left[\mathrm{Mg}_{2} \mathrm{SiO}_{4}\right]$ ).

La question centrale de l'évolution minérale est de comprendre comment une douzaine de minéraux, contenant 
environ dix éléments essentiels, ont évolué vers des milliers de minéraux portant soixante-douze éléments essentiels? Pour répondre à cette question, il a été proposé de diviser l'évolution minérale en différentes étapes, chacune augmentant la diversité minérale ${ }^{7}$.

$\mathrm{Au}$ début, des «poussières » présolaires condensées en gouttelettes, les chondres, suivies de leur agrégation forment les premiers corps sources de météorites appelées chondrites. Le matériel chondritique non altéré contient environ soixante minéraux réfractaires différents qui représentent le point de départ de l'évolution minérale de toutes les planètes rocheuses. Les chondrites se sont agrégées par une attraction gravitationnelle formant des «planétésimaux ». La masse croissante de ces corps rocheux fit qu'une évolution thermique se mit en place avec la formation d'achondrites : à ce stade environ 250 " nouveaux " minéraux se sont formés. Parmi eux, des silicates plus complexes ainsi que des phases carbonatées sont apparus.

À la suite de l'accrétion et de la différenciation planétaires, l'évolution minérale d'une planète rocheuse comme la Terre dépend d'abord d'une séquence de processus géochimiques et pétrologiques, comme le volcanisme et le dégazage, la cristallisation fractionnée, les réactions d'assimilation, le métamorphisme régional et de contact, la tectonique des plaques et les interactions fluide-roche. Sur la Terre, alors que les premiers terrains granitiques apparaissent, les pegmatites concentrent des éléments " incompatibles » rares. Ainsi, peuvent se former des nouvelles espèces minérales riches de béryllium, de bore, de lithium, de tantale, d'étain et d'ura- nium. Avec la formation de l'atmosphère, l'eau est apparue et de nouveaux processus de formation de minéraux ont été provoqués par des interactions fluide-roche. De vastes dépôts de minéraux hydrothermaux, de vastes terrains métamorphiques et des minéraux de haute pression sont formés ${ }^{8}$.

\section{Croissance et morphologie minérale}

Comme tous les systèmes naturels, les minéraux naissent, grandissent et évoluent en fonction du milieu environnant (espèces chimiques disponibles et conditions d'espace, de pression et de température). Ils se transforment ensuite et se modifient jusqu'à ce qu'ils soient entièrement recyclés, généralement pour former d'autres espèces minérales. Tout cela se produit en temps géologique (des centaines de milliers, voire des millions d'années), une échelle qui n'est pas comparable au temps de l'horloge qui régit la vie humaine. La première vitrine de l'alcôve 2 explique ce concept via des inclusions minérales (par exemple, des inclusions fluides dans un cristal d'halite), des zonations minérales (tourmalines) ou des croisements parallèles de la même espèce minérale comme dans le cas $\mathrm{du}$ « quartz sceptre » où un cristal hyalin (non coloré) est encapuchonné par une améthyste. Ces exemples donnent l'idée de la « temporalité » de la cristallisation. Les mêmes espèces minérales peuvent aussi croître avec des morphologies différentes en fonction de l'espace disponible. Une vitrine présente une sélection de morphologies « curieuses », principalement liées à différents environnements de cristallisation, telles que des concré- 
tions stalactitiques de rhodocrosite, de malachite et de smithsonite, de gerbes de natrolite ou d'améthyste et bien d'autres.

Les concepts fondamentaux de la cristallographie jusqu'à ceux de la symétrie morphologique et structurelle sont présentés à travers leur développement historique dans une quatrième vitrine. Sont présentés quelques instruments scientifiques $\mathrm{du} \mathrm{xVIII}^{\mathrm{e}}$ siècle appartenant aux " pères de la cristallographie ", comme le goniomètre de Haüy ou des modèles de cristaux de la collection Romé de l'Isle. Les échantillons minéraux illustrent la symétrie de chacun des sept systèmes cristallins de cristaux.

\section{La couleur des minéraux}

Jaune, bleu, rose, orange... toutes les couleurs sont présentes dans le monde minéral. Jusqu'au début du xIX siècle, la couleur était une caractéristique importante d'identification des minéraux. Aujourd'hui, ce critère n'est pas déterminant car le nombre d'espèces augmente considérablement et les mêmes espèces minérales peuvent présenter différentes couleurs. En outre, la plupart des minéraux sont naturellement incolores. L'origine de la couleur dans un minéral peut être à la fois chimique et physique.

À la base des phénomènes chimiques, les éléments chimiques, appelés " chromophores ", entrent dans la structure cristalline comme constituants ou impuretés. Dans le premier cas, une espèce minérale présente toujours la même couleur idiochromatique (malachite, azurite, cinabre, or et crocoite). Dans le deuxième cas, l'impureté provoque une couleur dite allochromatique. Par exemple, les traces de titane ou de manganèse dans le quartz le rendent rose; la présence de $\mathrm{Fe}^{3+}$ provoque la couleur jaune dans le quartz variété citrine; le $\mathrm{Fe}^{2+}$ est responsable de la couleur bleue du béryl variété aiguemarine et le $\mathrm{Mn}^{3+}$ pour le béryl rouge. La même impureté chimique peut générer des couleurs variées dans différents minéraux, car la couleur dépend de l'interaction entre l'impureté et la structure atomique environnante. L'allochromatisme peut également être associé à des défauts physiques en raison de l'absence accidentelle d'un cation ou d'un anion.

Dans la troisième alcôve, la diversité chromatique est illustrée par divers échantillons de minéraux et expliquée via un écran tactile. La fluorite, un minéral typiquement français et allochromatique, fait l'objet de la deuxième vitrine avec son défilé de couleurs.

Certains minéraux idiochromatiques conservent leur couleur lorsqu'ils sont broyés; dans ce cas, leurs poudres peuvent être utilisées comme pigments, en particulier dans la peinture, comme le montre aux visiteurs un dispositif combinant spécimens, pigments et iconographie. Des exemples de minéraux célèbres pour leur utilisation comme pigments sont présentés, tels l'or jaune par l'orpiment, le vermillon par le cinabre, l'hématite pour l'ocre rouge, la goethite pour l'ocre jaune, la malachite et la lazurite pour le vert et bleu.

\section{Minéraux et jeux de lumière}

Qu'elle soit réfléchie, réfractée, diffractée, réémise, absorbée, la lumière interagit de plusieurs façons avec les minéraux. On observe une grande diversité dans l'apparence des minéraux; des phénomènes étonnants se produisent. 
La couleur de certains minéraux peut être "pseudo-chromatique », ce qui s'explique par des phénomènes optiques dérivant de l'interaction entre la lumière et les caractéristiques physiques telles que les inclusions, la texture, et la structure cristallographique propre à chaque espèce minérale. Le phénomène connu comme opalescence est typique de l'opale noble.

L'iridescence et le pléochroïsme sont deux autres exemples de phénomènes optiques où la couleur du minéral change selon l'angle de vue ou l'orientation de la lumière incidente. La transparence et la luminosité sont deux propriétés qui dépendent de la façon dont la lumière passe ou est difractée par le minéral. La luminosité résulte de l'interaction de la lumière avec la surface du minéral.

La fluorescence et la phosphorescence sont des phénomènes luminescents causés par des rayonnements électromagnétiques (généralement des rayons ultraviolets) qui interagissent avec la structure électronique de certains minéraux. La lumière émise par l'échantillon s'arrête si le rayonnement excitant cesse, on dit que le minéral est fluorescent, un mot émanant de la fluorite, qui présente souvent ce phénomène. Les minéraux phosphorescents émettent également de la lumière après que le rayonnement se soit éteint. La phosphorescence ne dure généralement que quelques fractions de seconde et est difficilement observable à l'œil nu.

Sont illustrés dans la quatrième alcôve les phénomènes suivants : l'iridescence, le pléochroïsme (à l'aide d'un dispositif qui démontre la dépendance de la couleur à partir de l'orientation de la source lumineuse), la fluorescence (les miné- raux sont éclairés par la lumière blanche et UV, en séquence), la transparence et différents types de lustre (résineux, vitreux, métallique...).

\section{Pierres précieuses et pierres fines}

Émeraude, saphir, zircon, diamant, spinelle... les gemmes fascinent les hommes depuis des millénaires. Les spécimens les plus surprenants de la collection de pierres précieuses du Muséum sont exposés dans la cinquième alcôve, en spécimens bruts, pierres taillées, objets sculptés, pièces ouvragées. L'accent est mis sur les gemmes historiques de la collection, ayant notamment appartenu à la Couronne de France. Un théâtre optique raconte ainsi l'histoire du grand diamant bleu découvert en Inde, acheté par Louis XIV, volé pendant la Révolution. Les minéralogistes du Muséum ont récemment prouvé que le célèbre diamant Hope (conservé au Smithsonian Museum de Washington) a été taillé dans ce diamant bleu9.

Quatre pierres précieuses (diamant, rubis, saphir et émeraude) sont distinguées des «pierres fines " (topaze, grenat, opale, quartz, etc.). Leur valeur dépend beaucoup de la couleur, de la dureté, mais aussi d'autres critères tels que la limpidité, le poids (exprimé en carats) et la taille, sans parler de la rareté. Les diamants sont ainsi recherchés, ainsi que le souligne Gian Carlo Parodi ${ }^{10}$, pour l'absence de couleur : une légère teinte de jaune ou de brun les déprécie fortement. D'autre part, lorsque la couleur est forte, l'échantillon tombe dans la catégorie des diamants dits " colorés" et son prix augmente avec l'intensité de 
la couleur atteignant des sommes folles pour les nuances les plus rares : rouge, orange, bleu ou vert.

Le processus de taille des pierres précieuses, à la fois technique et artistique, a pour rôle de mettre en évidence les propriétés physiques et esthétiques des pierres, en particulier leur couleur et leur brillance. G. C. Parodi observe plusieurs aspects de la pierre selon sa taille. La coupe peut révéler des couleurs qui ne sont pas apparentes dans la matière première. Certains cristaux tels que l'émeraude, le rubis et le saphir, sont dichroïques et changent de couleur avec la direction d'observation. Dans ce cas, l'orientation de la coupe a un rôle fondamental sur la couleur qui apparaîtra. Un beau saphir bleu peut apparaître bleu-vert si sa coupe n'est pas correcte. D'autres pierres comme le diamant ont un indice de réfraction si élevé que la lumière doit rebondir à l'intérieur de la pierre avant qu'elle n'émerge vers l'œil. En fonction de cela, non seulement la pierre apparaît très brillante, mais sa couleur est beaucoup plus renforcée!

\section{Métaux précieux}

En quittant l'alcôve 5, les yeux du visiteur sont attirés par une vitrine dédiée aux métaux précieux. Parmi les minéraux, les métaux précieux et les pierres ornementales occupent une place spéciale en raison de leur utilisation par les hommes depuis des millénaires.

Pour les minéralogistes, les métaux précieux sont des espèces minérales appelées «éléments natifs ». L'or, l'argent et le platine natifs sont très appréciés en raison de leur caractère scientifique et esthétique unique.
L'or peut former des spécimens très fins, des masses compactes, des cristaux octaédriques - plus rarement cubiques - des veines, des filaments, ou encore des pépites. L'argent natif est principalement présent sous forme de filaments plus ou moins fins. Rarement, il peut former de grandes masses compactes, des cristaux aplatis ou généralement cubo-octaédriques. Le platine est principalement présent sous la forme de grains disséminés dans la roche qui le contient, habituellement microscopiques. Exceptionnellement, le platine peut former des pépites très recherchées par les collectionneurs. La Colombie et l'Oregon (États-Unis) ont produit des pépites de platine centimétriques. Les «monstres » proviennent tous de l'exploitation minière du xix ${ }^{\mathrm{e}}$ siècle en Sibérie. Le tsar Nicolas Ir a offert d'énormes pépites aux plus importants musées internationaux et trois d'entre elles enrichissent la collection du Muséum.

À l'exception de l'argent qui s'oxyde rapidement à l'air, les métaux rares ont une faible réactivité chimique, propriété contribuant à leur succès qui assure le maintien de la brillance. Ils sont généralement ductiles et ont un solide lustre métallique.

\section{Pierres ornementales}

Les pierres ornementales sont des roches ou minéraux, opaques ou translucides, utilisées dans les arts décoratifs : jaspe, lapis-lazuli, malachite, jade ou améthyste. La marqueterie de deux tables fabriquées, à Florence, par l'Opificio delle pietre dure au XVII ${ }^{\mathrm{e}}$ siècle, témoigne de cet usage dans la sixième alcôve. 
Certains minéraux et roches qui n'ont pas la rareté des gemmes, sont utilisés pour fabriquer ou décorer des objets d'apparat. Les agates et les jaspes, les porphyres et les granites, les calcaires plus ou moins colorés, les lapis-lazulis et les turquoises orientales, divers marbres et autres roches ornementales ont été employées dans des créations étonnantes depuis l'époque de la Rome impériale. Leur palette polychromatique suscitait l'imagination et poussait à l'ingéniosité pour les " assembler » dans des compositions variées. Cet art a connu son apogée à la cour des Médicis. L'Opificio, fondé en 1588 par le grandduc de Toscane, Ferdinand $\mathrm{I}^{\mathrm{er}}$, produisit pendant des décennies des œuvres lapidaires remarquables. Trois tables conservées au Muséum, décorées de motifs naturalistes, sont représentatives de ce type de production tellement apprécié qu'il fut brièvement implanté dans un atelier de la manufacture des Gobelins, à la demande de Louis XIV.

\section{Météorites}

Les météorites sont essentielles pour comprendre l'origine du soleil et de son cortège de planètes. Dans la dernière alcôve, les grands phénomènes qui ont été à la base de la formation du système solaire, sont expliqués à l'aide de trente météorites et deux écrans tactiles.

Le Muséum est dépositaire d'échantillons de météorites de la lune, de Mars et des principaux astéroïdes, petits corps planétaires qui peuplent la région entre Mars et Jupiter. Si les planètes ont considérablement évolué depuis leur formation, en même temps que le soleil, une fraction de ces petits corps - très rapide- ment refroidis - est restée " congelée ». Les météorites qui en dérivent contiennent des informations précieuses sur la genèse du système solaire, tandis que celles qui représentent des corps, ayant été géologiquement actifs, nous informent de l'évolution et de la nature interne des planètes. Ainsi, au-delà de la fascination qu'elles peuvent exercer par leur origine et leur arrivée spectaculaire, les météorites sont des objets d'investigation scientifique qui permettent de sonder l'espace aussi bien que le temps.

La minéralogie est " une science en mouvement » qui s'enrichit, chaque année, d'une centaine d'espèces nouvelles, la plupart rares et microscopiques, mais toutes ajoutant une brique à notre connaissance du laboratoire chimique unique connu sous le nom de Terre et, en partie, comme système solaire. En plus des échantillons de minéraux, le Muséum conserve depuis le XviII ${ }^{\mathrm{e}}$ siècle, l'une des plus grandes collections de " types " de minéraux dans le monde (plus de 300 des quelques 5000 espèces minérales officiellement acceptées par la Commission ad hoc de l'Association internationale de la minéralogie [IMA]). Ces échantillons de référence constituent le noyau des collections. Leur aspect esthétique n'est parfois pas spectaculaire et, pourtant, perçus dans une immuabilité apparente, beaucoup de ces échantillons sont des encyclopédies ouvertes sur l'histoire de notre planète.

C.F. 


\section{Notes}

1. Chapitre 2 du catalogue de l'exposition Cristaux géants. Minéraux précieux, rédigé par Henri-Jean Schubnel, ancien directeur de la galerie de minéralogie, Paris, Muséum national d'histoire naturelle, 1987.

2. Plusieurs des objets cités sont reproduits dans l'ouvrage collectif Trésors de la terre, Paris, Muséum national d'histoire naturelle, 2014, utile pour approfondir les divers thèmes de l'exposition; voir aussi Daniel Alcouffe, Les gemmes de la Couronne, Paris, Réunion des musées nationaux, 2001, Stéphane CAstelluccio, Les meubles de pierres dures de Louis XIV et l'atelier des Gobelins, Paris, Éditions Faton, 2007, Bernard Morel, Les joyaux de la Couronne de France, Paris, Fonds Mercator, Albin Michel, 1988.

3. J. A. Hugard, Muséum d'histoire naturelle de Paris. Galerie de minéralogie et de géologie. Description des collections..., Paris, Hachette, 1855.

4. H.-J. Schubnel, Cristaux géants. Minéraux précieux, op.cit., chapitre 4.

5. J. P. LoRAND, « Les cristaux géants", in Trésors de la terre, op. cit., p. 100.

6. R. M. Hazen, D. Papineau, W. Beeker, R. T. Downs, J. M. Ferry, T. J. MaC CoY, D. A. SvERJENSKY, H. YANG, «Évolution minérale», American Mineralogist, 93, 1693-1720, 2008.

7. Ibid.

8. Cristiano Ferraris, "La géodiversité », in Trésors de la terre, op. cit., p. 80-83.

9. François Farges, Thierry Piantanida, Le Diamant bleu, Paris, Michel Lafon, 2010.

10. François Farges, Gian Carlo PARodi, « Les minéraux précieux ", in Trésors de la terre, op. cit., p. 116. 